\title{
Intracranial Primary Malignant Solitary Fibrous Tumor/Hemangiopericytoma Masquerading as Meningioma: Report of a Rare Case
}

This article was published in the following Dove Press journal: International Journal of General Medicine

\author{
Zhixiang Sun ${ }^{1} *$ \\ Feng $\mathrm{Li}^{2}, *$ \\ Xintao Cai ${ }^{1}$ \\ Zhiquan Jiang'
}

'Departments of Neurosurgery, The First Affiliated Hospital of Bengbu Medical College, Bengbu 233000, People's Republic of China; ${ }^{2}$ Department of Radiation Oncology, The First Affiliated Hospital of Bengbu Medical College, Bengbu 233000, People's Republic of China

*These authors contributed equally to this work
Correspondence: Zhiquan Jiang Department of Neurosurgery, The First Affiliated Hospital of Bengbu Medical College, 287 Changhuai Road, Bengbu, Anhui 233004, People's Republic of China Tel +86-1396607597|

Email bbjiangzhq@I63.com
Background: Intracranial solitary fibrous tumors/hemangiopericytomas (SFTs/HPCs) are rare spindle cell tumors originating from interstitial tissue that are usually benign. Primary malignant intracranial SFTs/HPCs are extremely rare. Here we describe a case of malignant intracranial SFT/HPC.

Case Presentation: A 59-year-old woman presented with a space-occupying lesion in the left cerebellar tentorium. Based on imaging findings, we made a preoperative diagnosis of meningioma. During the operation, we found that the tumor had an abnormally rich blood supply and could not be completely removed. Histologic findings, immunophenotype (positive for cluster of differentiation $[C D] 34$, CD99, signal transducer and activator of transcription 6, and B cell lymphoma 2), and a Ki-67 proliferative index of $20-30 \%$ for the primary tumor were typical of malignant intracranial SFT/HPC. The tumors showed high sensitivity to radiotherapy and the residual tumor was significantly reduced after intensity-modulated radiation therapy. The patient has had no neurologic symptoms and no recurrence of the tumor in 2 years of follow-up.

Conclusion: Intracranial SFTs/HPCs are extremely rare. Radiologic examination before the operation is helpful for making a definite diagnosis and judging tumor grade; STAT6 immunohistochemistry is a sensitive alternative diagnostic method. Adjuvant radiotherapy is effective in cases of incomplete resection, and strict follow-up is essential in order to monitor for possible recurrence.

Keywords: intracranial, solitary fibrous tumor, malignant, radiation therapy

\section{Background}

Solitary fibrous tumors (SFTs) and hemangiopericytomas (HPCs) are rare spindle cell tumors of mesenchymal origin. ${ }^{1}$ The incidence of SFTs/HPCs involving the central nervous system (CNS) is low; the first case was only reported in $1996 .{ }^{2}$ SFTs and HPCs in the CNS have overlapping histologic and immunohistochemical features. ${ }^{3,4}$ In 2016 , the World Health Organization (WHO) combined SFTs and HPCs as a single clinical entity that can be categorized as 3 grades (I-III) according to a specific set of features. ${ }^{5}$ Most SFTs/HPCs of the CNS are benign lesions; malignant tumors are extremely rare. Here we report a case of primary malignant intracranial SFTs/HPCs originating from the tentorium cerebelli and mimicking meningioma.

\section{Case Presentation}

A 59-year-old woman presented at our hospital with dizziness and nausea that had gradually worsened over the previous month. Neurologic examination showed no 
abnormalities, but magnetic resonance imaging (MRI) revealed a well-defined mass in the left cerebellum and tentorium cerebelli measuring $31 \times 34 \times 42 \mathrm{~mm}$ in size. The T1- and T2-weighted images of the tumor were isointense, with large zones of edema surrounding the tumor; additionally, the tumor showed uneven enhancement (Figure 1A). Diffusion-weighted imaging showed a homogeneous signal, which was diminished at the tumor boundary. Head computed tomographic angiography showed clear tumor labeling. During the operation, the tumor was found to be tough, grayred, with a clear boundary and abundant blood supply. As it was located close to the transverse and sigmoid sinuses, only partial resection was performed. The residual tumor after the operation (Figure 1B) was treated with intensity-modulated radiation therapy (64 Gy, 32 times). Craniocerebral MRI performed 2 months later showed that the tumor was significantly smaller than before the operation (Figure 1C). The patient's symptoms and neurologic status also improved after surgical treatment. Regular follow-up examinations in the 2 years since have shown that the patient is free of disease, with no sign of tumor recurrence or metastasis.

Histopathologic analysis of the tumor showed an abundance of typical "staghorn" vascularization in the tissue; and there was considerable hyalinization in the blood vessel wall. A large number of fusiform tumor cells arranged in bundles were seen between blood vessels, and mitotic bodies were clearly visible ( $\geq 5$ mitoses per 10 high-power fields). The extracellular matrix was composed of bright eosinophilic collagen bands (Figure 2A and B), and the Ki-67 labeling index reached $20 \%$ in local hot spots. Immunohistochemical analysis revealed positivity for cluster of differentiation (CD)34 (Figure 3A), CD99, signal transducer and activator of transcription (STAT)6 (Figure 3B), and B cell lymphoma (BCL)-2 (Figure 3C); and negative staining for smooth muscle actin and desmin.

\section{Discussion and Conclusions}

SFTs/HPCs are rare spindle cell neoplasms of mesenchymal origin. CNS SFTs/HPCs account for $0.09 \%$ of all meningeal tumors; ${ }^{6}$ most occur in the fifth decade, without obvious sex differences. ${ }^{7}$ Tumors originate from the dura mater similar to meningioma and can occur in the tentorium and sickle and on the convex surface of the brain. There are no specific clinical symptoms until the tumor reaches a certain size or invades brain areas that produce detectable effects or have functional consequences.

Most SFTs grow slowly, but low-grade SFTs/HPCs can progress to higher-grade tumors. ${ }^{8}$ It is especially important to assess the biological behavior of SFTs/HPCs by imaging before the operation and to distinguish malignant SFT from benign lesions such as meningioma. The blood supply of SFTs/HPCs is abundant, and some cases have reported a dual blood supply of internal carotid artery and external carotid artery. Computed tomographic angiography is helpful for auxiliary diagnosis. Some cases show uneven high and low signals on T2-weighted images that appear as typical black-and-white or yin-yang signs. Cells in low-signal areas are sparse and enriched in collagen fiber bundles, with strong contrast enhancement; meanwhile, cells in high-signal areas are dense, and the

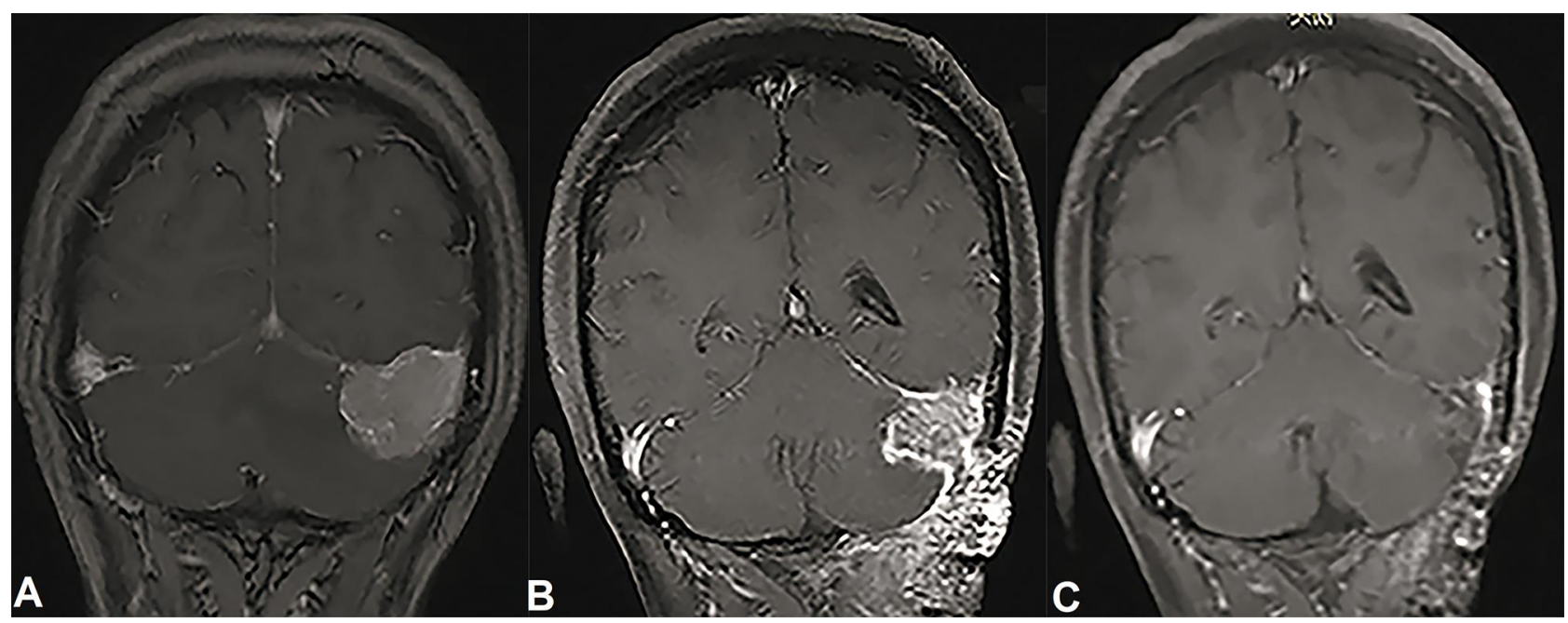

Figure I MRI of the patient with intracranial SFT/HPC. (A) MRI scan revealing tumors uneven enhancement, with large zones of edema around the tumors. (B and $\mathbf{C}$ ) Postoperative residual tumor (B) with a significantly reduced signal after 2 months of radiotherapy (C). 

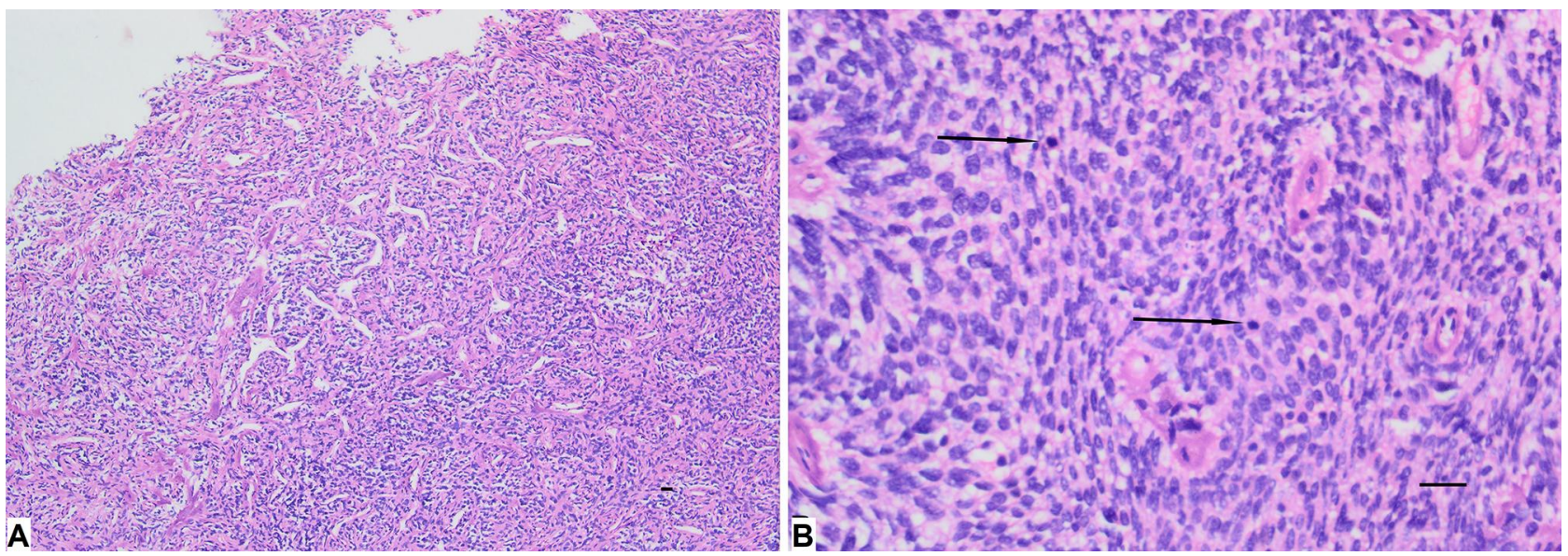

Figure 2 Hematoxylin and eosin staining of the SFT/HPC specimen. (A) An abundance of blood vessels was observed in tumor tissue, with an typical "staghorn" vascularization and a large number of fusiform tumor cells arranged in bundles between blood vessels. Magnification: 100x; scale bar: $100 \mu \mathrm{m}$. (B) Mitotic bodies were clearly visible (black arrow; $\geq 5$ mitoses per 10 high-power fields). Magnification: 400x; scale bar: $100 \mu \mathrm{m}$.

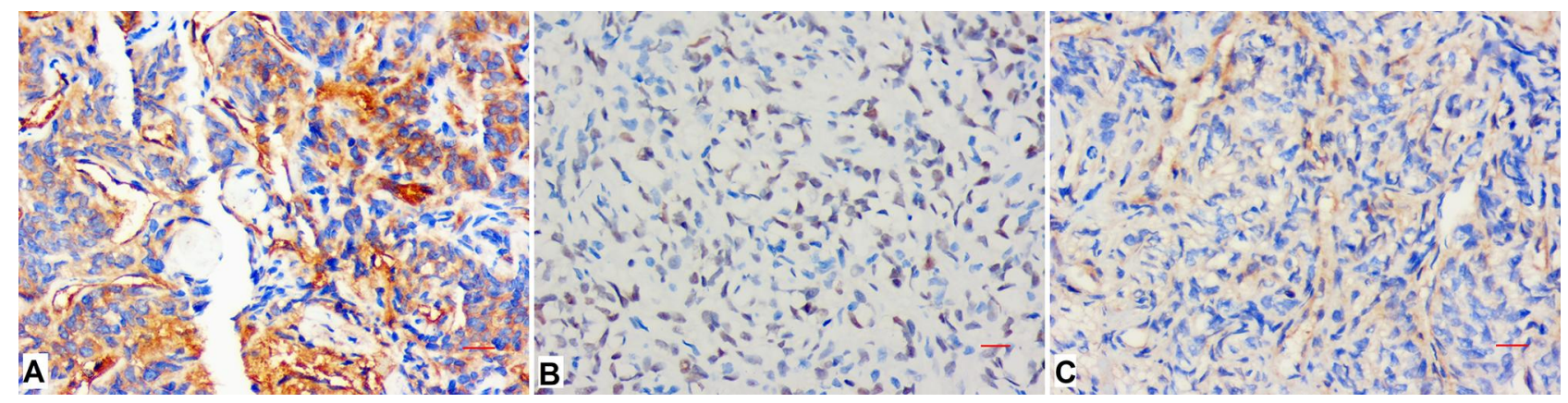

Figure 3 Immunohistochemical analysis of the SFT/HPC specimen. (A-C) with immunopositivity for CD34 (A), STAT6 (B), and BCL-2 (C). Magnification: 400x; scale bar: $100 \mu \mathrm{m}$.

enhancement effect is relatively weak. ${ }^{9,10}$ Skull erosion adjacent to intracranial SFTs of meningeal origin was found in $44.4 \%$ of cases $(4 / 9)$ in one study, ${ }^{11}$ in contrast to the typical bone thickening observed near meningioma. Additionally, compared to meningioma SFTs/HPCs are more prone to necrosis, cystic degeneration, and areas of signal void. Vascular ghosts and peritumoral edema were more clearly observed by MRI in high-grade (WHO grade III) than low-grade (WHO grades I and II) SFTs/HPCs, ${ }^{12}$ which was supported by our observations.

The diagnosis of intracranial SFTs/HPCs ultimately depends on pathologic and immunohistochemical findings. SFT and HPC were previously considered as distinct pathologic entities; however, there is increasing evidence that they share histologic features, immunohistochemical markers, and chromosomal abnormalities such as inversion at $12 \mathrm{q} 13$ and fusion of NGFI-A-binding protein $(N A B) 2$ and STAT6 genes. $^{3,4,13,14}$ According to the 2016 WHO classification of CNS tumors, meningeal SFTs and HPCs are interstitial tumors which assigned to three grades based on specific features. Grade I tumors have relatively low cellularity and are spindle cell lesions with so-called modeless structure that were previously classified as SFTs. Grade II tumors have more cells and less collagen; the cells are enlarged and surrounded by staghorn-shaped vasculature and are CNS HPCs. If there are $\geq 5$ mitoses per 10 high-power fields, the tumor is classified as grade III, which is associated with recurrence and metastasis. ${ }^{5}$ NAB2-STAT6 gene fusion is considered as a pathologic molecular feature of SFT. Notably, STAT6 immunohistochemistry was shown to be a sensitive and specific surrogate for identifying SFTs/HPCs. ${ }^{4}$ Similarly, detection of STAT6 nuclear expression is recommended to confirm the diagnosis of SFT/HPC as per the 2016 WHO guidelines. ${ }^{5}$ According to statistics, the specificity and sensitivity of nuclear STAT6 for SFT/HPC were $100 \%$ and $96.6 \%$, respectively. ${ }^{15}$ The sensitivity and specificity of ALDH1 for diagnosing SFT were $84 \%$ and $98.8 \%$, respectively, whereas its sensitivity for meningioma was just $1.2 \% .{ }^{16}$ Additionally, 
$77.9 \%$ of SFTs are CD34-positive compared to $6.4 \%$ of meningiomas. Interestingly, CD34 expression rates in $\mathrm{WHO}$ grades I, II, and III tumors were $90.2 \%, 76.3 \%$, and $67.7 \%$, respectively, ${ }^{15}$ revealing an inverse relationship between SFT/HPC grade and CD34 level; this trend has not been observed for any other marker. SFTs/HPCs are generally positive for CD99, vimentin, and BCL-2 but negative for epithelial membrane antigen and S-100, as in our case. Based on the results of the histopathologic analysis, our case was diagnosed as WHO grade III SFT/HPC.

Surgical resection is the first-choice treatment for SFT/ $\mathrm{HPC}$, and gross total resection is the most critical factor for tumor control. However, this is technically difficult to achieve with most tumors of a higher grade which invasion of surrounding important anatomical structures such as arteries, venous sinuses, nerves, etc., as in our case. In such instances, subtotal resection plus adjuvant radiotherapy is a good choice. ${ }^{17}$ Total resection and adjuvant radiotherapy can prolong the time to extracranial metastasis in patients, ${ }^{18}$ while radical resection combined with postoperative radiotherapy can increase overall survival and prolong the time to recurrence. ${ }^{19}$ The tumor in our patient showed good sensitivity to radiotherapy, and there has been no recurrence in the 2 years of follow-up. However, given the degree of malignancy, close monitoring and long-term follow-up are needed.

In summary, intracranial SFTs/HPCs are an extremely rare tumor. Radiologic examination before the operation is helpful for making a definite diagnosis and judging tumor grade. STAT6 immunohistochemistry is a sensitive alternative diagnostic method. Adjuvant radiotherapy is effective for malignant tumors that cannot be completely resected. Finally, strict follow-up is essential in order to monitor for possible recurrence.

\section{Data Sharing Statement}

The data that support the findings of this study are available on request from the corresponding author, Zhiquan Jiang.

\section{Ethics and Consent Statement}

Based on the regulations of the department of research of the Bengbu Medical College, institutional review board approval is not required for case reports.

\section{Consent for Publication}

Written informed consent has been provided by the patient to have the case details and any accompanying images published.

\section{Author Contributions}

All authors contributed to data analysis, drafting or revising the article, have agreed on the journal to which the article will be submitted, gave final approval of the version to be published, and agree to be accountable for all aspects of the work.

\section{Funding}

The authors declared that this case has received no financial support.

\section{Disclosure}

The authors declare that they have no competing interests.

\section{References}

1. Bisceglia M, Galliani C, Giannatempo G, et al. Solitary fibrous tumor of the central nervous system: A 15-year literature survey of 220 cases (August 1996-July 2011). Adv Anat Pathol. 2011;18:356-392. doi:10.1097/PAP.0b013e318229c004

2. Carneiro SS, Scheithauer BW, Nascimento AG, Hirose T, Davis DH. Solitary fibrous tumor of the meninges: a lesion distinct from fibrous meningioma. A clinicopathologic and immunohistochemical study. Am J Clin Pathol. 1996;106:217-224. doi:10.1093/ajcp/106.2.217

3. Bouvier C, Métellus P, de Paula AM, et al. Solitary fibrous tumors and hemangiopericytomas of the meninges: overlapping pathological features and common prognostic factors suggest the same spectrum of tumors. Brain Pathol. 2012;22:511-521. doi:10.1111/j.17503639.2011.00552.x

4. Schweizer L, Koelsche C, Sahm F, et al. Meningeal hemangiopericytoma and solitary fibrous tumors carry the NAB2-STAT6 fusion and can be diagnosed by nuclear expression of STAT6 protein. Acta Neuropathol. 2013;125:651-658. doi:10.1007/s00401-013-1117-6

5. Louis DN, Perry A, Reifenberger G, et al. The 2016 World Health Organization classification of tumors of the central nervous system: a summary. Acta Neuropathol. 2016;131:803-820. doi:10.1007/ s00401-016-1545-1

6. Gengler C, Guillou L. Solitary fibrous tumour and haemangiopericytoma: evolution of a concept. Histopathology. 2006;48:63-74.

7. England DM, Hochholzer L, MacCarthy MJ. Localized benign and malignant fibrous tumors of the pleura. A clinicopathologic review of 223 cases. Am J Surg Pathol. 1989;13:640-658. doi:10.1097/ 00000478-198908000-00003

8. Apra C, Mokhtari K, Cornu P, Peyre M, Kalamarides M. Intracranial solitary fibrous tumors/hemangiopericytomas: first report of malignant progression. J Neurosurg. 2018;128:1719-1724. doi:10.3171/ 2017.1.JNS162593

9. Frazier AA. The Yin and Yang of solitary fibrous tumor. Radiographics. 2014;34:294. doi:10.1148/rg.342144003

10. Weon YC, Kim EY, Kim HJ, Byun HS, Park K, Kim JH. Intracranial solitary fibrous tumors: imaging findings in 6 consecutive patients. AJNR Am J Neuroradiol. 2007;28:1466-1469. doi:10.3174/ajnr. A0609

11. Clarençon F, Bonneville F, Rousseau A, et al. Intracranial solitary fibrous tumor: imaging findings. Eur J Radiol. 2011;80:387-394. doi:10.1016/j.ejrad.2010.02.016

12. Zhou JL, Liu JL, Zhang J, Zhang M. Thirty-nine cases of intracranial hemangiopericytoma and anaplastic hemangiopericytoma: a retrospective review of MRI features and pathological findings. Eur J Radiol. 2012;81:3504-3510. doi:10.1016/j.ejrad.2012.04.034 
13. Robinson DR, Wu YM, Kalyana-Sundaram S, et al. Identification of recurrent NAB2-STAT6 gene fusions in solitary fibrous tumor by integrative sequencing. Nat Genet. 2013;45:180-185. doi:10.1038/ng.2509

14. Chmielecki J, Crago AM, Rosenberg M, et al. Whole-exome sequencing identifies a recurrent NAB2-STAT6 fusion in solitary fibrous tumors. Nat Genet. 2013;45:131-132. doi:10.1038/ng.2522

15. Yamashita D, Suehiro S, Kohno S, et al. Intracranial anaplastic solitary fibrous tumor/hemangiopericytoma: immunohistochemical markers for definitive diagnosis. Neurosurg Rev. 2020. doi:10.1007/ s10143-020-01348-6

16. Bouvier C, Bertucci F, Metellus P, et al. ALDH1 is an immunohistochemical diagnostic marker for solitary fibrous tumours and haemangiopericytomas of the meninges emerging from gene profiling study. Acta Neuropathol Commun. 2013;1:10. doi:10.1186/20515960-1-10
17. Combs SE, Thilmann C, Debus J, Schulz-Ertner D. Precision radiotherapy for hemangiopericytomas of the central nervous system. Cancer. 2005;104:2457-2465. doi:10.1002/cncr.21448

18. Schiariti M, Goetz P, El-Maghraby H, Tailor J, Kitchen N. Hemangiopericytoma: long-term outcome revisited. Clinical article. J Neurosurg. 2011;114:747-755. doi:10.3171/2010.6.JNS091660

19. Chen LF, Yang Y, Yu XG, Gui QP, Xu BN, Zhou DB. Multimodal treatment and management strategies for intracranial hemangiopericytoma. J Clin Neurosci. 2015;22:718-725. doi:10.1016/j. jocn.2014.11.011

\section{Publish your work in this journal}

The International Journal of General Medicine is an international, peer-reviewed open-access journal that focuses on general and internal medicine, pathogenesis, epidemiology, diagnosis, monitoring and treatment protocols. The journal is characterized by the rapid reporting of reviews, original research and clinical studies across all disease areas. The manuscript management system is completely online and includes a very quick and fair peer-review system, which is all easy to use. Visit http://www.dovepress.com/ testimonials.php to read real quotes from published authors.

Submit your manuscript here: https://www.dovepress.com/international-journal-of-general-medicine-journal 